\title{
Usefulness of point shear wave elastography to assess the effects of extracorporeal shockwaves on spastic muscles in children with cerebral palsy: an uncontrolled experimental study
}

\author{
B. Corrado1, M. Albano, M.G. Caprio², C. Di Luise ${ }^{1}$, M. Sansone ${ }^{3}$, V. Servodidio¹, \\ S. Russo ${ }^{1}$, G. Vallone ${ }^{4}$, E.A. Vola ${ }^{4}$, C. Servodio lammarrone ${ }^{1}$ \\ 1 Department of Public Health, University of Naples Federico II, Naples, Italy \\ 2 Institute of Biostructure and Bioimaging, National Research Council of Italy, Naples, Italy \\ ${ }^{3}$ Department of Electrical Engineering and Information Technology (DIETI), University of Naples Federico II, \\ Naples, Italy \\ ${ }^{4}$ Department of Advanced Biomedical Sciences, University of Naples Federico II, Naples, Italy
}

\author{
CORRESPONDING AUTHOR \\ Bruno Corrado \\ University of Naples Federico II \\ Via Pansini 5 \\ 80131 Napoli, Italy \\ Phone: +390817462796 \\ E-mail: bruno.corrado@unina.it
}

DOI

10.32098/mltj.01.2019.04

\begin{abstract}
SUMMARY
Background. Muscle spasticity causes disability in children with cerebral palsy. Extracorporeal shockwave therapy has proved to be effective in managing hypertonic muscles. Shear wave elastography can objectively assess tissue elasticity properties. The aim of the present study was to 1) evaluate the effectiveness of defocused extracorporeal shockwaves in reducing soleus muscle contracture in a group of children with cerebral palsy by using point shear wave elastography, and 2) analyse the correlation between the Modified Ashworth Scale and ultrasound elasticity index. Methods. Children with spastic cerebral palsy $(n=21)$ were administered extracorporeal shockwave therapy ( 5 sessions at weekly interval, 500 defocused shockwaves per session, $0.25 \mathrm{~mJ} / \mathrm{mm}^{2}, 3 \mathrm{~Hz}$ ). The effects were measured by means of point shear wave elastography 1, 3, and 6 months after therapy. Results. Linear mixed effects modelling showed significant reduction of muscle stiffness, with the predicted greatest effect at the 13 th week after completion of treatment. Analysis of covariance confirmed the correlation between the Modified Ashworth Scale and ultrasound elasticity index. Conclusions. Point shear wave elastography confirmed the effectiveness of defocused extracorporeal shockwaves in reducing muscles contracture in children with cerebral palsy. We recommend the use of this technique for the clinical assessment of spastic patients.
\end{abstract}

\section{KEYWORDS}

cerebral palsy; muscle spasticity; elasticity imaging techniques; extracorporeal shockwave therapy

\section{BACKGROUND}

Cerebral palsy $(\mathrm{CP})$ comprises a group of neurological disorders resulting from malformation in the developing brain or nonprogressive brain injury occurring during or soon after birth. CP is the most common childhood disability, with a reported prevalence of 2-3 per 1000 live births (1). Physical disability in CP is mainly due to motor impairment, and manifests as poor muscle control, weakness, hypertonicity, spasticity, and balance disorders. The motor impairment frequently leads to contractures of muscle-tendon units with consequent reduction of the range of motion (RoM) of joints. Contracture most commonly affects the ankle plantar flexors, leading to equinus foot deformity. Contractures are not solely due to muscle overactivation; increased intrinsic passive stiffness of the tissue also contributes (2). Thus, increased muscle stiffness in CP has two components: active and passive. Active (neural) muscle stiffness is mainly related to spasticity, which is due to velocity-dependent exces- 
sive muscle activation resulting from hyperexcitability of the stretch reflex. By contrast, passive (non-neural) muscle stiffness is due to the viscoelastic properties of muscles (3). On clinical examination, active muscle stiffness is exhibited when the muscle is contracting, while passive muscle stiffness is exhibited when myofibers are not actively contracting. The source of active muscle stiffness is the contractile cells, while the source of passive muscle stiffness is the connective tissue and extracellular matrix.

While muscle contracture may play a limited positive role in CP by compensating for muscle weakness, it has an overall negative effect on mobility control and function. Contractures may also affect musculoskeletal development and lead to skeletal deformities, joint dislocations, and scoliosis.

Treatment of muscle contractures can be a major challenge in children with CP. The treatment strategy depends on the nature of stiffness (active, passive, or mixed) and on the degree and location of functional impairment. Treatment options include oral medication (baclofen, diazepam, clonazepam, dantrolene, and tizanidine), physical and occupational therapy, splinting and casting, chemodenervation with botulinum toxin or phenol, selective dorsal rhizotomy, intrathecal baclofen, and orthopaedic surgery.

A new treatment option could be extracorporeal shockwave therapy (ESWT), which has been found to be highly effective in chronic soft tissue injuries and certain bone conditions (4). ESWT can be applied using focused or defocused extracorporeal shockwaves. The two types differ in focal zone localization, inside the human body and inside the therapy head of the device, respectively. Defocused shockwaves exert their effect at the body surface and are preferred for treatment of muscular disorders. In recent years, defocused shock wave therapy has been considered as an effective treatment modality in regeneration medicine (5). However, the underlying mechanisms of beneficial effect of defocused shock waves are not yet completely understood. Previous studies suggested that upregulated neovascularization (6), neuroregeneration (7), enzymatic activities (8), anti-inflammatory effect (9), and recruitment of endogenous mesenchymal stromal cells (MSCs) (10) might be involved in the process. Over the last few years several studies have found ESWT to be effective for treating muscle contractures in children with $\mathrm{CP}$ via its neuromodulatory and rheological effects (11-15). However, until now only subjective or operator-dependent assessment tools have been used in order to measure the effects of ESWT on spastic muscles. Here, it is necessary to clarify that radial pressure waves are another kind of waves which definitely differ from extracorporeal shock waves in generation, mechanism of action and indications. Moreover, also radial pressure waves are used to treat muscle contractures.
Measurement of muscle stiffness is difficult. Ordinal scales such as the Ashworth Scale (AS) and the Modified Ashworth Scale (MAS) are easy to apply but lack reliability and do not measure the passive component of muscle stiffness (16). Recent advances in ultrasound (US) elastography techniques have now made direct quantification of muscle stiffness possible. US elastography is a noninvasive imaging technique that measures tissue displacement (i.e., strain) in response to the application of a given force. There are several US elastography methods available, depending on how the force is applied; two commonly used methods are transient elastography (TE), which uses mechanical external vibration, and shear wave elastography (SWE), which uses acoustic radiation force impulse (ARFI). All elastography methods allow to measure the Strain Ratio (SR), which is used when the force applied in elastography is not known. SR is calculated by dividing the average strain in the region of interest (ROI) by the average strain in the reference normal tissue. SR is a dimensionless quantity and is generally expressed as a fraction with a number between 0 and 1 . The higher SR is, the more stiff the tissue. In SWE, ARFI creates shear waves that travel perpendicularly through the tissue. These waves can be tracked for a limited distance. The velocity of the propagating shear waves is more in harder than in softer tissue, and this property is made use of for evaluating tissue stiffness (17). The relationship between the applied force and the resulting strain, which is expressed as either shear wave velocity $(\mathrm{m} / \mathrm{s})$ or as the elasticity index $(\mathrm{EI})$, also called Young's modulus $(\mathrm{kPa})$, quantifies tissue elasticity (17). The higher the EI, the more stress is needed to create the same amount of strain; an idealized rigid body would have an infinite EI. The visual representation of tissue elasticity and the possibility to perform quantitative assessment of recorded tissue elasticity allows to further categorize SWE into Point Shear Wave Elastography (P-SWE) and 2D Shear Wave Elastography (2D-SWE).

$\mathrm{P}-\mathrm{SWE}$ is a recently introduced form of SWE. It can be incorporated into high-end ultrasound equipment, allowing B-mode ultrasound elastography measurements. The technique has many advantages: it is an operator-independent, relatively reproducible, quantitative method for the evaluation of muscle tissue properties (18). The usefulness of P-SWE for measurement of muscle stiffness in children with CP has been demonstrated by Vola and co-authors in 2018 (19).

In this experimental study we aimed to 1) evaluate the effects of extracorporeal defocused shockwaves on soleus muscle contracture in children with hemiplegic $\mathrm{CP}$ by using two clinical measurements (MAS and ankle pRoM) and two P-SWE interpretation parameters (SR and EI), and 2) analyse the correlation between MAS scores and EI. 


\section{MATERIALS AND METHODS}

A total of 21 children (15 male and 6 female; age range, 3-16 years) with hemiplegic CP were recruited from the Federico II University Outpatient Rehabilitation Centre of Naples, Italy.

In order to compute the sample size for a minimum power of 0.80 , we performed a simulation as suggested by Benjamin et al. (20). We repeated each simulation 5000 times per sample size. It turned out that a minimum of 4 subjects was sufficient for testing the hypothesis of parabolic trend with subject-dependent intercept, with respect to a constant trend. The sample size required to test the same hypothesis with respect to other trends (linear or parabolic, with subject-dependent coefficients) is 2 . In these last cases the power remains always higher than 0.9 .

The study was carried out in accordance with the 7th revision of the Declaration of Helsinki and informed consent for the processing of the personal data was provided in advance with appropriate, written documentation by parent/guardian/tutor. Privacy and confidentiality were completely guaranteed (21). Inclusion criteria were 1) verified diagnosis of $\mathrm{CP}$ with unilateral spastic equinus foot deformity; 2) walking ability, with or without aids; 3 ) ability to follow instructions; 4) dynamic ankle plantar flexion contractures (confirmed if foot equinus deformity was observed during ambulation); and 5) spasticity of plantar flexor muscles greater than grade 1 and up to grade 4 according to the MAS. The exclusion criteria were 1) previous surgery at the level of feet, ankles, or legs; 2) botulinum toxin injections in the preceding 6 months; 3 ) current treatment with spasticity medications or drugs which could influence muscle activity; 4) clinical signs of myopathy or neuropathy; 5) serious blood dyscrasia or clotting disorder; 6) infection or cancer at the site of therapy application.

We decided to analyse the effects of defocused shockwaves on the soleus because it is a monoarticular muscle and its sole function is plantar flexion. In contrast, the gastrocnemius, which is the other plantar flexion muscle, also contributes to knee flexion. So, with the knee flexed, the soleus muscle becomes the main plantar flexion muscle. Furthermore, due to its deep anatomical position the soleus is more suitable than the gastrocnemius muscle for ultrasound evaluation with a convex transducer.

Clinical and ultrasonographic assessments were performed before the first treatment session (T0), and immediately (T1-5th week), 1 month (T2-9th week), 3 months (T3-17th week), and 6 months (T4-29th week) after the last treatment session. The first evaluation was in June-July 2017 and the last one in January-February 2018. At each follow-up, the children underwent a comprehensive clinical examination (including maximum ankle dorsiflexion measurement and
MAS) by a physiatrist specialized in paediatric rehabilitation and an ultrasound examination (P-SWE) performed by a radiologist with expertise in musculoskeletal ultrasound. In each patient the same physiatrist applied the shockwave therapy and performed the clinical evaluation.

Ultrasonographic and clinical evaluations were performed always under the same environmental conditions, that is to say at a temperature between 20 and $22{ }^{\circ} \mathrm{C}$ (climate-controlled room) and in the morning.

Maximum ankle dorsiflexion ( $\mathrm{pRoM}$ ) was assessed using a goniometer. Measurement was performed with the patient in the supine position, with the knee flexed at $90^{\circ}$. Spasticity of the soleus was measured using MAS.

Each child received one ESWT session per week for 5 consecutive weeks. Therapy was performed with an electromagnetic shockwave device (Duolith ${ }^{\circledR}$ SD1, Storz Medical AG, Taegerwillen, Switzerland) that produces defocused shockwaves by means of the C-ACTOR ${ }^{\circledR}$ II handpiece. Defocused shockwaves have short wavelengths, with their focal zone inside the therapy head of the device. Defocused shockwaves exert their effects at the body surface and are preferred for treatment of muscular disorders. A total of 500 defocused shockwaves were used to treat each hypertonic soleus muscle. For each treatment an energy level of $0.25 \mathrm{~mJ} / \mathrm{mm}^{2}$ at a frequency of $3 \mathrm{~Hz}$ was applied. ESWT was repeatedly performed at the same anatomical site as the P-SWE evaluation, i.e., at the proximal one-third of the soleus on a longitudinal line running from a point midway between medial and lateral epicondyles of the femur to the calcaneal tuberosity. During therapy the child was positioned prone, with the legs stretched out on the table. An ultrasound gel was applied on the skin at the site of placement of the head of the device. No anaesthesia or analgesia was used.

All children received physiotherapy concurrently with ESWT (60-minute sessions, every other day). Physiotherapy was carried out by licensed physical therapists and consisted of positioning, stretching exercises, massages, proprioceptive neuromuscular facilitation (Bobath technique) and sensory integration therapy. However, it should be pointed out that physical therapy was already performed before ESWT and went on after the treatment as well.

The radiologist performed P-SWE using a Samsung RS80A ultrasound scanner with Prestige equipment (Samsung Medison Co. Ltd., Seoul, Korea), interfaced with a convex array transducer (CA1-7; Samsung Medison Co. Ltd., Seoul, Korea), and equipped with Smart Shear Wave Elastography (S-SWE) software (figure 1). The S-SWE method had a unique performance index, Reliability Measurement Index (RMI), which is calculated by the weighted sum of the residual of the wave equation and the magnitude of the 


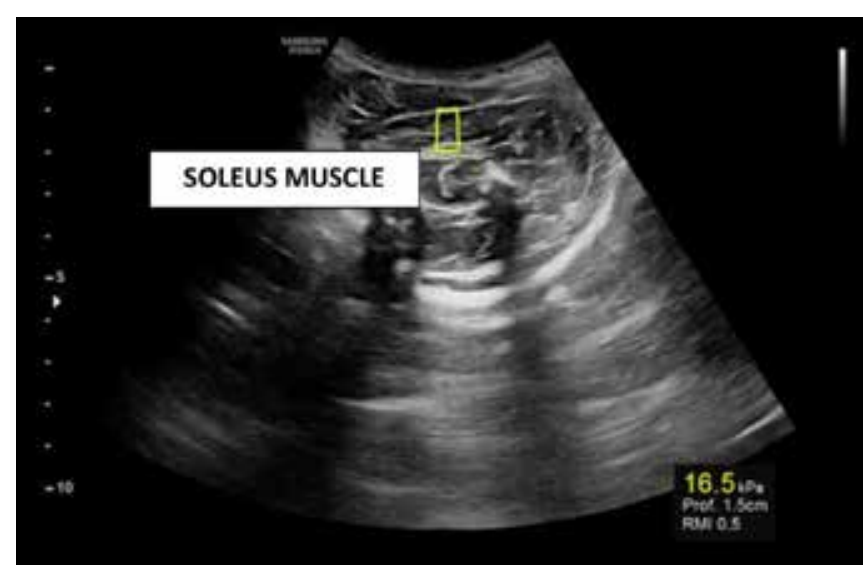

Figure 1 - Point Shear Wave Elastography.

shear wave. RMI ranging from $0.0-1.0$, where 0.4 or higher is considered as acceptable whilst 1.0 is considered a very high value of RMI, and strongly correlates with reproducible measurements, according to the manufacturer. The proposed index is utilized to filter out unreliable measurements and results in performance improvement of shear wave elastography. Although several sources of system-dependent bias exist for each type of shear wave elastography, a recent phantom study has compared the S-SWE to other novel shear wave methods, demonstrating a similar and excellent inter- and intra-observer repeatability (22).

During examination the child was in the prone position, with the feet dangling over the edge of the examination table. Ultrasound measurement was performed over the middle third of the soleus muscle, where the muscle bulk is greatest and no fascial borders, tendons, and major blood vessels are present. The transducer was aligned transversally to the direction of the muscle fibers. B-mode imaging was used to confirm correct placement of the transducer. The transducer was held in place with minimal pressure on the skin.

\section{STATISTICAL METHODS}

Statistical analysis was performed using R (http:// www.R-project.org/). Linear mixed effects (LME) modelling was used to analyse the changes over time of SR, EI, and ankle pRoM (23). This method is suitable when there are repeated measures on the same patient. The classical analysis of variance (ANOVA) cannot be applied because of the correlation that exists between measurements in the same patient. A preliminary analysis showed that a second-order polynomial (such as $\mathrm{y}(\mathrm{t})=\mathrm{A} \times \mathrm{t}^{2}+\mathrm{B} \times \mathrm{t}+\mathrm{C}$ ) might be an appropriate fitting per each patient. Specifically, we tested the hypothesis that all patients could be described by polynomials, differing only for the constant $\mathrm{C}$ (that might depend on the initial value of the stiffness for that patient). Therefore, we assumed $\mathrm{A}$ and $\mathrm{B}$ as fixed effects and $\mathrm{C}$ as random effect; thus, the complete model can be described by the equation $\mathrm{y}(\mathrm{j}, \mathrm{k})=\mathrm{A} \times \mathrm{k}^{2}+\mathrm{B} \times \mathrm{k}+\mathrm{C}(\mathrm{j})+\mathrm{e}(\mathrm{j}, \mathrm{k})$, where $y(j, k)$ is the measurement on the $\mathrm{j}$ subject at time $\mathrm{k} ; \mathrm{C}(\mathrm{j})$ is the constant coefficient of the $\mathrm{j}$-th patient while e $(\mathrm{j}, \mathrm{k})$ is the error term at time $\mathrm{k}$ for the $\mathrm{j}$-th patient. We assumed that $\mathrm{C}(\mathrm{j})$ is normally distributed with variance $\Sigma \mathrm{C}^{2}$ and diagonal covariance matrix; $e(j, \mathrm{k})$ is normal with diagonal covariance matrix and variance sigma^ 2 . This model has been tested against two other models: $\mathrm{A}$ fixed, $\mathrm{B}$ and $\mathrm{C}$ random with diagonal covariance matrix; $\mathrm{A}, \mathrm{B}$, and $\mathrm{C}$ random with diagonal covariance matrix. To investigate the relationship between EI and MAS the repeated-measures correlation approach was used (24). The classical Pearson correlation coefficient cannot be applied, because the condition of data independence is not satisfied and within-subject correlation must be taken into account. The repeated-measures correlation method is based on analysis of covariance (ANCOVA) and provides the best fit for each subject, with parallel lines and variable intercepts. Currently an implementation of this approach is contained in the rmcorr package in $\mathrm{R}$. The repeated-measures correlation coefficient was calculated, with the confidence interval (calculated with bootstrap on 1000 repetitions) and the $\mathrm{p}$ value.

\section{RESULTS}

Figures 2 and 3 show the EI vs. time relationship, represented as mean and individual measurements respectively. As figure 2 shows, minimum stiffness was measured at the 9th week after completion of treatment, and then EI mean value progressively returned to pre-treatment status. The beneficial effects of ESWT disappeared at the 24th week approximately. Figure 3 shows that LME modelling gives a reasonable approximation of the measured data. A formal analysis of LME gave a residual standard deviation of 0.482 ; the coefficients of the second-order polynomial were 7.95 , $-0.278,0.0104$ (mean of the random C, fixed B and A, respectively), while the standard deviation of the random effect $(\mathrm{C})$ was 1.49. The comparison of this model with the other two gave no statistically significant improvement $(\mathrm{p}>$ 0.05 in both cases); therefore, we decided to use the random $\mathrm{C}$ because of its simplicity. Diagnostic analysis of residuals did not reveal serious lack of normality, which was verified also by a formal Shapiro test of normality ( $p>0.05)$ or heteroscedasticity confirmed by a Levene test $(\mathrm{p}>0.05)$. Using the values of second-order coefficients a minimum stiffness can be predicted at about the 13th week. Similar observations can be made about SR vs. time and pRoM vs. 


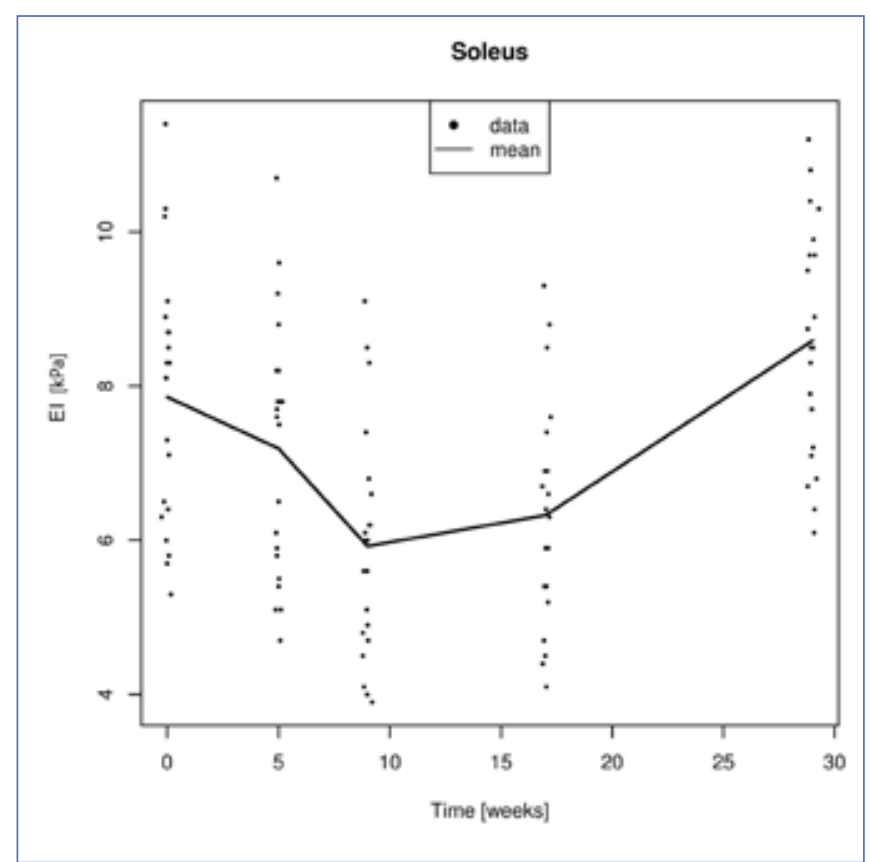

Figure 2 - Soleus muscle Elasticity Index (EI) expressed in kilopascal as a function of time expressed in weeks. Points represent data from each patient while solid line represents mean.

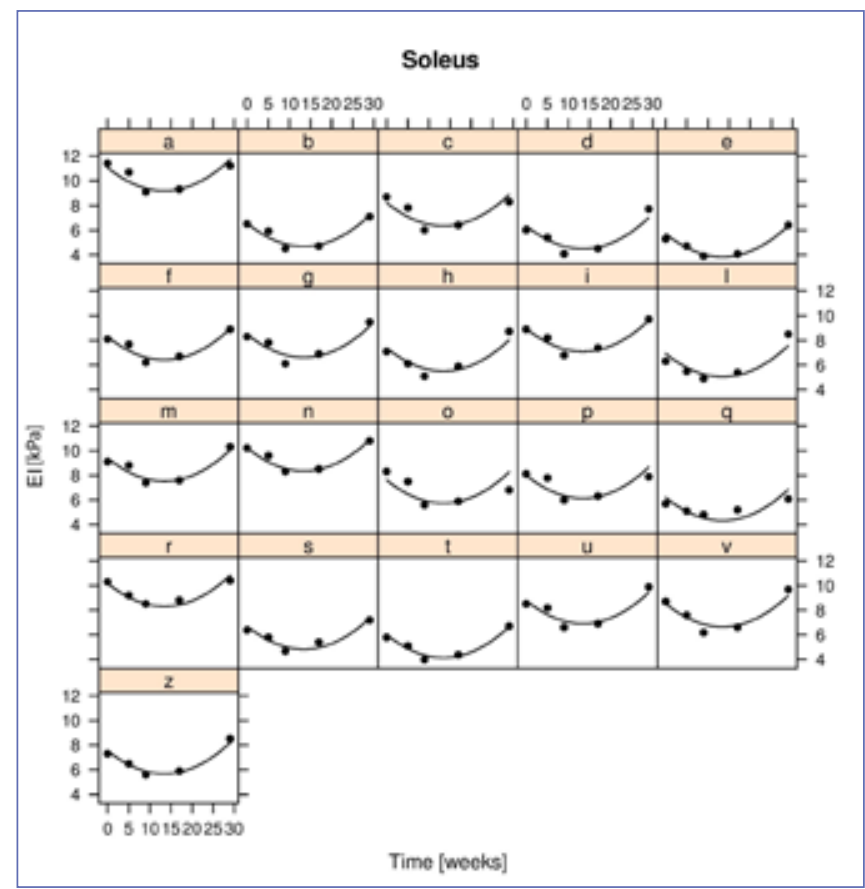

Figure 3 - Soleus muscle Elasticity Index (EI) expressed in kilopascal as a function of time expressed in weeks. To each patient is reported its own panel. time relationships; in both cases we observed a parabolic trend with minimum values at the 9th week follow-up and a gradual return to the pre-ESWT measures throughout the 15 following weeks. LME modelling allows to predict minimum values at the 13th week also with regard to soleus SR and ankle pROM. Figure 4 shows the scatterplot of EI vs. MAS for each patient in separate panels and highlights the close correlation between the two parameters.

\section{DISCUSSION}

P-SWE confirmed that extracorporeal shockwaves can reduce muscle contracture in children with CP. Post ESWT there was statistically significant improvement in SR and EI. The statistical model (LME) showed that the lowest level of muscle stiffness was attained in the 9th week after completion of treatment, and that the effect disappeared by the 24th week. The model also predicted that the effects of ESWT would be highest at the 13th week. Furthermore, the repeated-measures correlation analysis found positive correlation between MAS score and EI.

Our findings should be now compared with those of similar previous studies which consider the same type of shock waves, namely the extracorporeal shock waves, and the same kind of disorder, that is to say CP. Papers about radi-

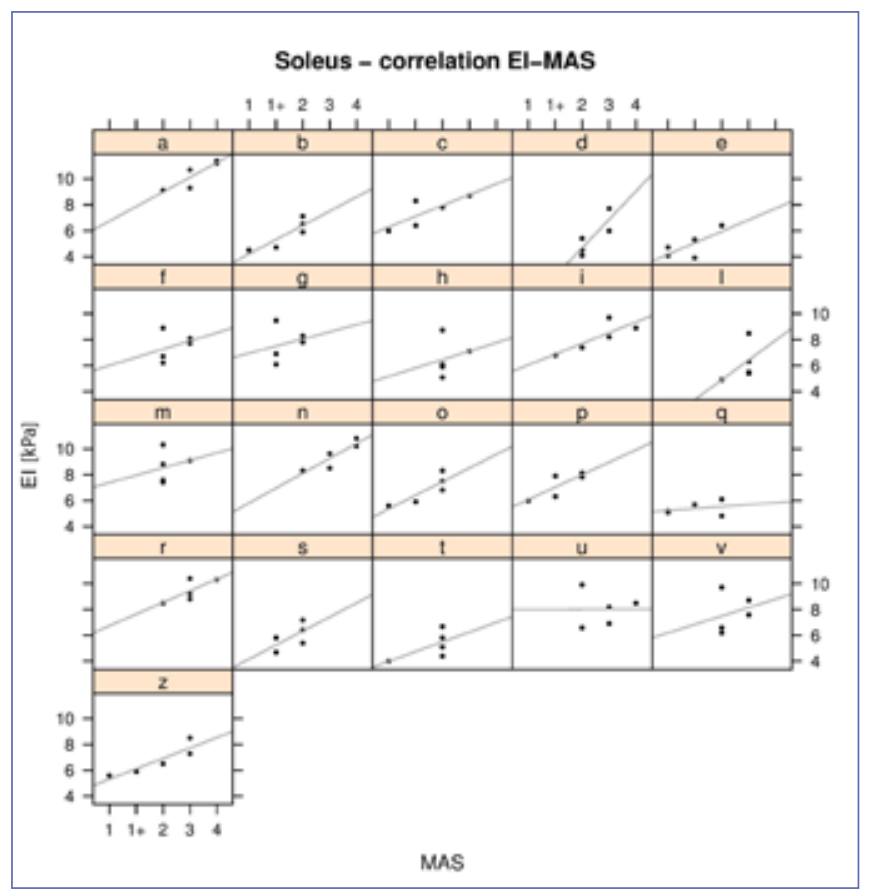

Figure 4 - Relation between soleus muscle Elasticity Index (El) expressed in kilopascal and soleus muscle MAS score. To each patient is reported its own panel.

Muscles, Ligaments and Tendons Journal 2019;9 (1) 
al waves or post-stroke spasticity were fully excluded from comparative analysis. It should be made clear our study was the first ever that evaluated the efficacy of defocused extracorporeal shockwaves on spastic muscles, whereas all previous studies have reported the effects of focused shockwaves. Focused and defocused extracorporeal shock waves share both physical basis and biological effects. However, it is well known that defocused shockwaves are the most suitable for treatment of muscle disorders, especially since they allow to treat a larger area at the same time. This is the reason why the energy level we used in this experimental study was definitely higher than that used by previous researchers. It is also necessary to state that the P-SWE method we used in this research, namely the S-SWE, has never been employed for the evaluation of ESWT effects on spastic muscles. S-SWE allows the most accurate measurements of tissue stiffness compared to other SWE methods, according to the manufacturer.

In the 2010 self-controlled trial by Amelio and Manganotti (15) a single active shockwave stimulation (1500 SW, energy level $0.030 \mathrm{~mJ} / \mathrm{mm}^{2}$, frequency not specified) produced a significant 4-week-long reduction in the hypertonia of the plantar flexors of 12 children with CP. Although their study included a control group, the small sample size, the shortterm follow-up (4 weeks), and the use of only subjective (MAS and ankle pRoM) or nonstandardized (baropodometry) measures of muscle stiffness represent important limitations compared to our research. In a 2014 study on a large group of 64 children with CP, Mirea et al. (12) showed that ESWT (3 sessions, $500 \mathrm{SW}$ per session, energy level $0.15 \mathrm{~mJ} / \mathrm{mm}^{2}$, frequency $10 \mathrm{~Hz}$ ) could decrease muscle spasticity at different anatomical sites. Although the sample size was large, the lack of a control group, the treatment of different muscles, the use of only subjective assessment tools (MAS, Gross Motor Function Classification System, Gross Motor Function Measure 66, and Questionnaire on Pain caused by spasticity), and the short-term follow-up are important weaknesses. El-Shamy et al. (14) in 2014 showed that ESWT (12 sessions at weekly intervals, $1500 \mathrm{SW}$, energy level $0.030 \mathrm{~mJ} / \mathrm{mm}^{2}$, frequency $5 \mathrm{~Hz}$ ) could improve plantar flexors spasticity and gait pattern in children with CP. The randomized controlled design and the use of gait analysis for assessment represent the main strengths of their study. Nevertheless, there were some important limitations, for example, the large number of ESWT sessions and the single follow-up at the end of the treatment. In 2015 Park and co-authors (11), for the first time, used ultrasonography to study the effectiveness of ESWT ( 1 vs. 3 sessions, 1500
SW per session, energy level $0.030 \mathrm{~mJ} / \mathrm{mm}^{2}$, frequency 4 $\mathrm{Hz}$ ) for reducing hypertonicity of the medial gastrocnemius in children with CP. Their case-control study had a relatively small sample size ( $\mathrm{n}=12$ ) and short follow-up (4 weeks). Moreover, they used the TE technique, which is operator dependent (because the stress applied can vary) and lacks precision in the color-coded graphic representation of stiffness. Picelli et al. (13) in 2017 proved that the combination of botulinum toxin serotype A (BoNT-A) and ESWT ( 1 session per week for 3 weeks, 1500 SW per session, energy level $0.030 \mathrm{~mJ} / \mathrm{mm}^{2}$, frequency $4 \mathrm{~Hz}$ ) is more effective than BoNT-A alone for treatment of spasticity of different muscles in a group of 10 children with CP. Follow-up lasted 4 weeks and assessment of the stiffness was with SWE. The randomized controlled design and the use of SWE are the main strengths of Picelli's study. However, the small sample size, the short-term follow up, the use of ESWT in association with BoNT-A, and the employment of SR (semi-quantitative assessment) as the only elastographic outcome represent important limitations compared to our study.

The main strengths of this study were the use of an operator-independent instrument (P-SWE) to measure muscle stiffness and the long-term follow-up. However, this study has two main limitations. First, the sample size was small (n = 25); nevertheless, it should be noted that it is the largest study to date using ultrasonography to measure the effect of ESWT on CP hypertonic muscles. Second, a causal relationship cannot be established because of the observational nature of the study. Performing clinical trials in children is difficult because of heterogeneity of the population and the particular ethical concerns in this age-group, such as protection against unknown risks and harms (25).

The present study has two important implications for clinicians. First, we have confirmed once again that ESWT (in particular defocused shockwaves) is effective for managing muscles contractures in children with CP. The maximal effect occurs at 13 weeks post treatment, after which the effects progressively decline until the 24th week. Thus, it may be reasonable to apply ESWT at least twice a year. Second, it is advisable to use an objective assessment tool, such US elastography, for clinical evaluation of muscle spasticity in children with CP. In particular, P-SWE should be used to measure the passive component of spastic muscle contracture (muscle fibrosis), which is barely assessed by MAS.

The authors are awaiting Ethical Committee approval for a randomized controlled trial, with a control group treated by sham ESWT. 


\section{REFERENCES}

1. Colver A, Fairhurst C, Pharoah PO. Cerebral palsy. Lancet 2014; 383(9924):1240-1249.

2. Mathewson MA, Lieber RL. Pathophysiology of muscle contractures in cerebral palsy. Phys Med Rehabil Clin N Am 2015; 26(1):57-67.

3. Norris JA, Cabrera MN, Smith TL, Reeves SH, Koman LA. Quantifying spasticity in a clinical setting. Biomed Sci Instrum 2007; 43:284-289.

4. Lama A, Santoro A, Corrado B, Pirozzi C, Paciello O, Pagano TB, Russo S, Calignano A, Mattace Raso G, Meli R. Extracorporeal shock waves alone or combined with raloxifene promote bone formation and suppress resorption in ovariectomized rats. PLoS One. 2017; 12(2):e0171276.

5. Kisch T, Wuerfel W, Forstmeier V et al. Repetitive shock wave therapy improves muscular microcirculation. J Sur Res 2016; 201(2):440-445.

6. Wang CJ, Wang FS, Yang KD et al. Shock wave therapy induces neovascularization at the tendon-bone junction. A study in rabbits. J Orthop Res 2003; 21(6):984-989.

7. Qiu X, Lin G, Xin Z et al. Effects of low-energy shockwave therapy on the erectile function and tissue of a diabetic rat model. J Sex Med 2013; 10(3):738-746.

8. Haupt G. Use of extracorporeal shock waves in the treatment of pseudarthrosis, tendinopathy and other orthopedic diseases. J Urol 1997; 158(1):4-11.

9. Veronesi F, Maglio M, Tschon M, Aldini NN, Fini M. Adipose-derived mesenchymal stem cells for cartilage tissue engineering: state-of-the-art in in vivo studies. J Biomed Mater Res A 2014; 102(7):2448-2466.

10. Jin Y, Xu L, Zhao Y, Wang M, Jin X, Zhang H. Endogenous Stem Cells Were Recruited by Defocused Low-Energy Shock Wave in Treating Diabetic Bladder Dysfunction. Stem Cell Rev 2017; 13(2):287-298.

11. Park DS, Kwon DR, Park GY, Lee MY. Therapeutic Effect of Extracorporeal Shock Wave Therapy According to Treatment Session on Gastrocnemius Muscle Spasticity in Children With Spastic Cerebral Palsy: A Pilot Study. Ann Rehabil Med 2015; 39(6):914-921.

12. Mirea A, Onose G, Padure L, Rosulescu E. Extracorporeal shockwave therapy (ESWT) benefits in spastic children with cerebral palsy (CP). J Med Life 2014; 7SpecNo. 3:127-132.

13. Picelli A, La Marchina E, Gajofatto F et al. Sonographic and clinical effects of botulinum toxin Type A combined with extracorporeal shock wave therapy on spastic muscles of children with cerebral palsy. Dev Neurorehabil 2017; 20(3):160-164.

14. El-Shamy SM, Eid MA, El-Banna MF. Effect of extracorporeal shock wave therapy on gait pattern in hemiplegic cerebral palsy: a randomized controlled trial. Am J Phys Med Rehabil 2014; 93(12):1065-1072.

15. Amelio E, Manganotti P. Effect of shock wave stimulation on hypertonic plantar flexor muscles in patients with cerebral palsy: a placebo-controlled study. Rehabil Med 2010; 42(4):339-343.

16. Mutlu A, Livanelioglu A, Gunel MK. Reliability of Ashworth and Modified Ashworth scales in children with spastic cerebral palsy. BMC Musculoskelet Disord. 2008; 9:44.

17. Gennisson JL, Deffieux T, Fink M, Tanter M. Ultrasound elastography: principles and techniques. Diagn Interv Imaging 2013; 94:487-495.

18. Arda K, Ciledag N, Aktas E, Aribas BK, Kose K. Quantitative assessment of normal soft-tissue elasticity using shearwave ultrasound elastography. AJR Am J Roentgenol 2011; 197(3):532-536.

19. Vola EA, Albano M, Di Luise C et al. Use of ultrasound to measure muscle stiffness in children with cerebral palsy. J Ultrasound 2018 Jul 20. doi: 10.1007/s40477-018-0313-6. [Epub ahead of print].

20. Arnold BF, Hogan DR, Colford JM Jr, Hubbard AE. Simulation methods to estimate design power: an overview for applied research. BMC Med Res Methodol 2011; 11:94.

21. Padulo J, Oliva F, Frizziero A, Maffulli N. Muscles, Ligaments and Tendons Journal - Basic principles and recommendations in clinical and field Science Research: 2016 Update. Muscles Ligaments Tendons J 2016; 6(1): 1-5.

22. Mulabecirovic A, Mjelle AB, Gilja OH, Vesterhus M, Havre RF. Repeatability of shear wave elastography in liver fibrosis phantoms-Evaluation of five different systems. PLoS ONE. 2018; 13(1):e0189671.

23. Venables WN, Ripley BD. Modern Applied Statistics with S. Fourth edition. 2002 Springer-Verlag, New York. ISBN 0-38795457-0.

24. Bakdash JZ, Marusich LR. Repeated Measures Correlation. Front Psychol 2017; 8:456.

25. Joseph PD, Craig JC, Caldwell PH. Clinical trials in children. Br J Clin Pharmacol. 2015;79(3):357-369. 